\title{
Trajectory-Based Takeoff Time Predictions Applied to Tactical Departure Scheduling: Concept Description, System Design, and Initial Observations
}

\author{
Shawn A. Engelland ${ }^{1}$ \\ NASA Ames Research Center, Fort Worth, TX, 76155 \\ Alan Capps ${ }^{2}$ \\ Mosaic ATM, Fort Worth, TX, 76155
}

\begin{abstract}
Current aircraft departure release times are based on manual estimates of aircraft takeoff times. Uncertainty in takeoff time estimates may result in missed opportunities to merge into constrained en route streams and lead to lost throughput. However, technology exists to improve takeoff time estimates by using the aircraft surface trajectory predictions that enable air traffic control tower (ATCT) decision support tools. NASA's Precision Departure Release Capability (PDRC) is designed to use automated surface trajectory-based takeoff time estimates to improve en route tactical departure scheduling. This is accomplished by integrating an ATCT decision support tool with an en route tactical departure scheduling decision support tool. The PDRC concept and prototype software have been developed, and an initial test was completed at air traffic control facilities in Dallas/Fort Worth. This paper describes the PDRC operational concept, system design, and initial observations.
\end{abstract}

\section{Nomenclature}

$\begin{array}{ll}\text { APREQ } & =\text { Approval Request }- \text { see CFR } \\ \text { ARTCC } & =\text { Air Route Traffic Control Center } \\ \text { ATCT } & =\text { Air Traffic Control Tower } \\ \text { CFR } & =\text { Call For Release } \\ \text { OFF } & =\text { aircraft takeoff time } \\ \text { TMC } & =\text { Traffic Management Coordinator } \\ \text { TMU } & =\text { Traffic Management Unit } \\ \text { TRACON } & =\text { Terminal RADAR Approach Control }\end{array}$

\section{Introduction}

GUTURE air traffic demands are expected to require a greater degree of integration among the automation Systems used to manage arrival, departure and surface traffic. The next generation air transportation system (NextGen) envisions Integrated Arrival/Departure/Surface (IADS) operations as described in the JPDO Integrated Work Plan $^{1}$ and in the FAA's NextGen Mid-Term Concept of Operations. ${ }^{2}$ Various NextGen concepts ${ }^{3,4}$ describe $^{3}$ IADS operations that feature a greater degree of automated coordination as traffic flows from one control domain to the next in the tactical air traffic control environment.

A logical first step towards the NextGen vision of fully-integrated arrival/departure/surface operations is to automate tactical scheduling of departure traffic that will join a constrained en route traffic flow. A commonly used tactical Traffic Management Initiative (TMI) is the Call For Release (CFR) procedure which is also known as the Approval Request (APREQ) procedure. CFR procedures vary from facility to facility; however, they generally require the Air Traffic Control Tower (ATCT) to request approval from the Air Route Traffic Control Center (ARTCC) prior to releasing departures destined to specified destinations. Earlier research ${ }^{6,7}$ at NASA Ames focused

\footnotetext{
${ }^{1}$ Aerospace Engineer, NASA/FAA North Texas Research Station, AIAA Senior Member.

${ }^{2}$ National Airspace System Engineer, NASA/FAA North Texas Research Station, AIAA Senior Member.
} 
on automating inter-facility coordination during CFR procedures. An FAA-led effort built on this work to develop and evaluate the Departure Flow Management prototype. ${ }^{8}$

Presently, en route tactical departure scheduling to meet CFR restrictions is often accomplished with the Traffic Management Advisor (TMA) decision support tool. A companion paper ${ }^{9}$ presents a characterization of current tactical departure scheduling based on analysis of more than one million flights from operational TMA data sets. This in-depth analysis identifies shortfalls in present-day tactical departure scheduling operations and suggests that the shortfalls can be remedied by reducing the uncertainty in CFR takeoff time predictions.

The Precision Departure Release Capability (PDRC) research activity combines the automated coordination demonstrated in the previous research with the use of surface trajectory-based takeoff (OFF) time predictions to improve en route tactical departure scheduling during CFR procedures. The PDRC concept and prototype software have been developed, and an initial test was recently completed at NASA's North Texas (NTX) Research Station in Dallas/Fort Worth (DFW). The test culminated in several opportunistic operational runs where PDRC times were used to schedule actual DFW departures. This paper describes the PDRC operational concept, system design and initial qualitative observations from the recent field test. Quantitative results are not yet complete, but they will include an analysis of OFF time predictions and an assessment of en route scheduling performance with particular attention to slot utilization and controller workload. Metrics for this quantitative analysis are developed and presented in the companion paper. ${ }^{9}$

\section{Concept Description}

The PDRC operational concept addresses shortfalls in present-day tactical departure scheduling operations. This section begins with a brief overview of tactical departure scheduling. The overview is followed by a description of the tactical departure scheduling situations for which PDRC is designed. This section concludes with a presentation of the PDRC operational concept.

\section{A. Tactical Departure Scheduling Overview}

Tactical departure scheduling is a process used by Traffic Management Coordinators (TMCs) to regulate air traffic flow to eliminate local demand/capacity imbalances and ensure local traffic management initiatives are achieved. Tactical departure scheduling is distinguished from strategic departure scheduling based upon scope (both temporal and geographic), precision requirements, and the decision support tools used. ${ }^{9}$ Strategic departure scheduling primarily uses the Traffic Flow Management (TFM) tool suite while tactical departure scheduling is typically accomplished with the Traffic Management Advisor (TMA) tool.

In 1996, NASA successfully demonstrated a prototype TMA system at Fort Worth ARTCC (ZFW) and DFW TRACON (D10). ${ }^{10}$ The NASA prototype TMA was primarily focused on arrival metering; however, it did include a nascent internal departure scheduling capability to support departures from within the ARTCC that were destined to the TMA-metered airport. The FAA's subsequent TMA development effort improved internal departure scheduling. Adjacent center metering was introduced in 2003 when ZFW controllers began metering Houston-bound traffic using times computed by the Houston ARTCC (ZHU) TMA system. TMA adjacent center metering capabilities enable "internal" departure scheduling beyond the arrival airport's home ARTCC. In 2006 the FAA added the En Route Departure Capability (EDC) to TMA building upon NASA's Multi-Center TMA research, ${ }^{11}$ and further enhancing TMA's tactical departure scheduling capabilities. EDC is commonly used to apply miles-in-trail restrictions and to regulate departures into constrained airspace. As of January 2011 the FAA has deployed TMA to 80 operational facilities: 20 ARTCCs, 31 TRACONS, 28 ATCTs and the ATCSCC.

Thus, TMA includes two distinct functions for tactical departure scheduling. These decision support tool functions are closely associated with the outbound and inbound tactical departure situations that form the backdrop for the PDRC operational concept. Outbound tactical departure scheduling uses TMA's EDC function to tactically schedule flights departing from airports within the TMA system's home ARTCC outbound into constrained en route streams or airspace. Whether the flight's destination is in the adjacent ARTCC or across the country, this situation is considered tactical because EDC is being used to address local demand/capacity imbalances and because scheduling is to a meter point in the home ARTCC. Inbound tactical departure scheduling uses the TMA arrival metering "internal" departure function to schedule departures into the inbound arrival streams of a TMA-metered airport. These inbound departures may originate from the TMA system's home ARTCC, another ARTCC, or even other countries (e.g. Canadian flights scheduled into New York ARTCC's TMA system via the international TFM interface). Examples of outbound and inbound tactical departure scheduling situations are described in the following section. 
The latest version (3.12.0) of TMA introduces a new tactical departure scheduling function called Coupled Scheduling. ${ }^{12}$ In simple terms, Coupled Scheduling will connect TMA EDC schedules produced in one ARTCC with TMA arrival metering schedules produced in an adjacent ARTCC. This new function will link outbound and inbound tactical departure scheduling. TMA 3.12.0 was deployed in the summer of 2011. The new Coupled Scheduling function will be selectively activated after key site checkout which began in August 2011.

\section{B. Tactical Departure Scheduling Situations}

PDRC is intended for use in tactical departure scheduling situations. During these situations, TMCs commonly employ the CFR procedure to address local demand/capacity imbalances and ensure local traffic management initiatives are satisfied. Note that the locally-implemented CFR procedure may be in response to a constraint elsewhere in the NAS that is propagated to create a local demand/capacity imbalance. The CFR procedure may be applied in both the outbound and inbound tactical departure scheduling modes.

The following discussion uses real-world situations to illustrate how these tactical departure scheduling modes are used. The examples feature ZFW and DFW because this is the locale for the initial PDRC field tests. These situations are, however, representative of tactical departure scheduling situations found throughout the National Airspace System (NAS).

\section{Outbound Tactical Departure Scheduling}

Figure 1 shows an example of an outbound tactical departure scheduling situation. The figure depicts the southeastern portion of the United States. State boundaries are shown in grey while ARTCC boundaries are shown in purple. The green arrows depict a cross-country overhead stream of traffic destined to Atlanta's HartsfieldJackson International Airport (ATL). The overhead stream transits ZFW airspace crossing directly over DFW and continues to ATL via the Meridian (MEI) fix. The gold arrow depicts the ascending flight of a DFW departure that must merge with the overhead stream of ATL-bound traffic.

The DFW departures merge with the overhead stream at the MEI arc $(155 \mathrm{~nm}$ radial distance west from the MEI fix) located on the extreme eastern edge of ZFW. This arc serves as a meter reference point for TMA's EDC function. TMA EDC uses four-dimensional (4D) trajectory synthesis including aircraft performance models and current forecast winds to calculate an OFF time that will enable the aircraft to rendezvous with the identified slot at the meter

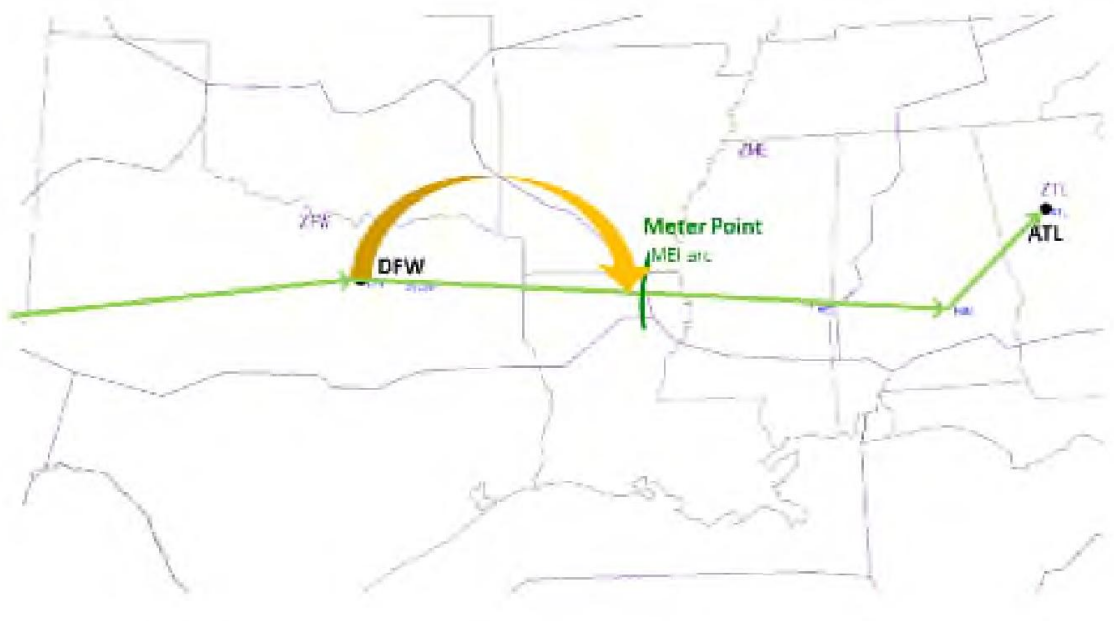
point - the MEI arc in this example.

The ATL flow shown here represents a fairly common CFR situation for ZFW; however, ZFW uses EDC for outbound tactical departure scheduling to Houston-area airports approximately four times more often than for ATL.

\section{Inbound Tactical Departure Scheduling}

Figure 2 shows an example of an inbound tactical departure scheduling situation. In this case the green arrows show various traffic streams destined to Houston's George Bush Intercontinental Airport (IAH). These streams pass through ZFW airspace and converge at the TORNN meter arc (73nm radial distance northwest from the RIICE fix) which is situated at the southern boundary of ZFW. In TMA terminology, the TORNN arc is an "outer metering arc." TMA distributes the delay an aircraft must absorb to various meter reference points (i.e. metering arcs and fixes) situated along the arrival path. Metering at the TORNN arc is actually performed by ZFW controllers using information provided by the ZHU TMA system via adjacent center metering infrastructure. 
As before a gold arrow depicts a DFW departure that must merge with the constrained overhead stream going to IAH. Traffic in these flows is being regulated by the arrival TMA system at Houston ARTCC (ZHU). The blue arc shows the ZHU arrival TMA scheduling freeze horizon for these IAH-bound traffic streams. Arrival TMA schedules must be frozen a certain distance from the metering point to provide the sector controller with a workable schedule. Since DFW lies inside this freeze horizon, DFW departures to IAH will be competing with overhead traffic for which the arrival TMA metering times have already been established.

The challenge depicted in Fig. 2 is addressed by TMA's inbound tactical departure scheduling capability. In this situation ZFW TMCs are able to schedule IAH-bound DFW departures into the ZHU TMA system prior to departure. As part of this pre-departure scheduling process, a release time is computed for the DFW departure and communicated to the ATCT via the CFR procedure.

Inbound tactical departure scheduling is not unique to ZFW and ZHU. Figure 3 presents a survey of inbound tactical departure scheduling operations. The base image is courtesy of the FAA and depicts TMA adjacent center metering installations in the NAS. The base image shows the 20 ARTCCs as green "puzzle piece" polygons. The small blue and black labels and dashed lines in the base image are not relevant to this discussion.

Operational TMA data has been analyzed ${ }^{9}$ to identify sites where inbound tactical departure scheduling operations occur. The survey results are presented via overlay symbols in Fig. 3. The ARTCCs have been labeled with round yellow "Os" and square orange "Ds". The "O" label indicates ARTCCs that schedule their departures

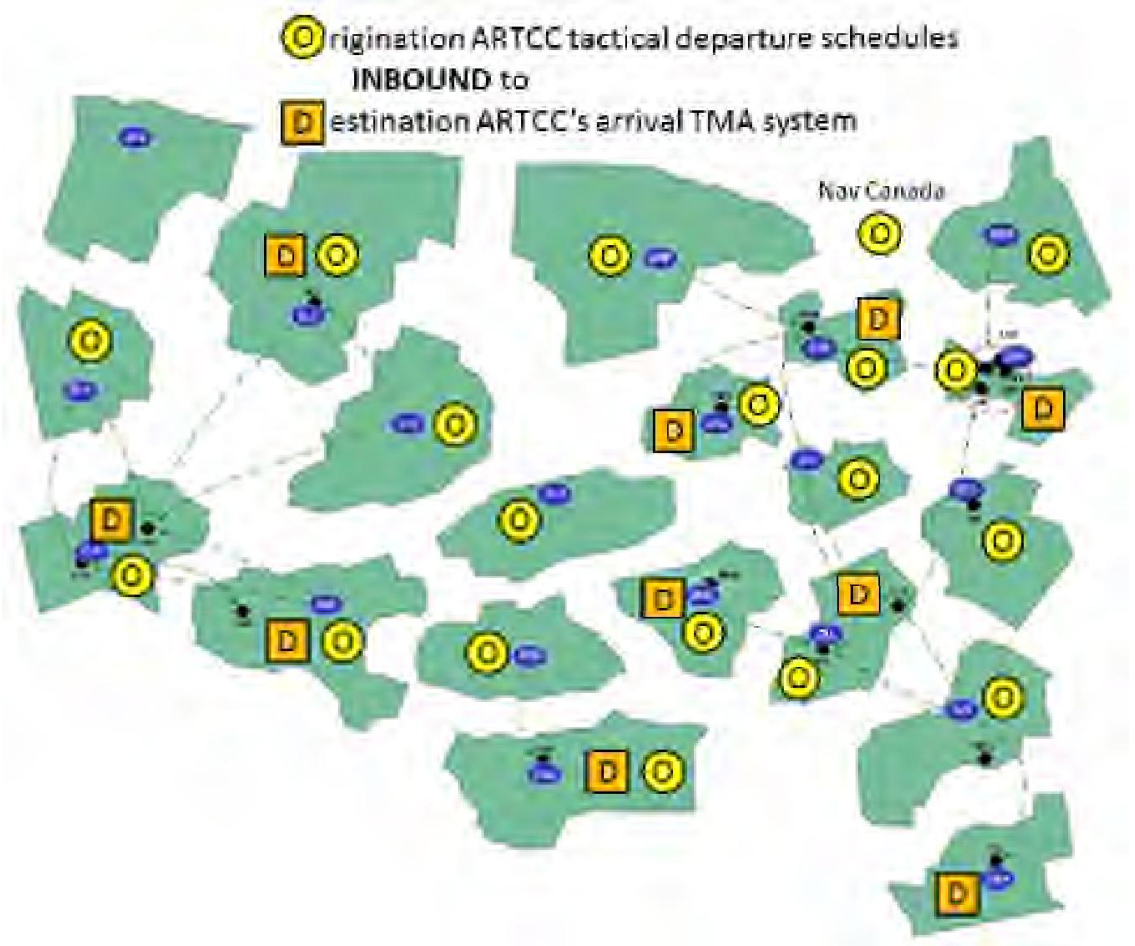
into the arrival TMA system of one or more adjacent ARTCCs. The "D" label identifies ARTCC's that permit adjacent facilities to schedule departures into their arrival TMA system. Note, there is one "O" label in the northeast that is not associated with an ARTCC. This symbol represents inbound tactical departure scheduling from Nav Canada to New York ARTCC (ZNY). Figure 3 shows that 18 ARTCCs (plus Nav Canada) schedule departures into another ARTCC's TMA system while 10 ARTCCs receive inbound tactical departure schedules from other facilities.

Figure 3. Survey of ARTCCs using TMA inbound tactical departure scheduling. 


\section{Concept Overview}

Figure 4 provides a high-level overview of the PDRC operational concept. This figure is applicable to both the outbound and inbound tactical departure scheduling situations described in the preceding section. The right side of the figure depicts departure traffic operating under the CFR procedure where departures must be merged into constrained en route traffic flows. The left side of the figure shows the PDRC decision support tools used for tactical departure scheduling.

The upper portion of the figure depicts a traffic stream in the en route domain that is under a CFR constraint. The ARTCC uses a tactical departure scheduling decision support tool to schedule departures into this constrained overhead stream. Ascent modeling in the en route decision support tool enables precise time-based scheduling and de-confliction at the meter point. The modeled ascent trajectory is illustrated by the gold line in Fig. 4 .

The lower portion of Fig. 4 depicts the ATCT environment where a NextGen surface trajectory-based decision support tool is in use. NextGen surface trajectory-based operations are enabled by a surface surveillance system and air carrier data sharing which provides intent and status information (i.e. gate assignments, estimated and actual pushback times, etc.). The surface trajectories computed and used by this decision support tool are represented by the blue and red lines in this figure.

PDRC focuses on the automated communication and use of surface trajectory-based OFF time predictions for tactical departure scheduling in CFR situations. In present-day operations, OFF time prediction and communication is manual. PDRC communication is illustrated by the double-headed yellow arrow on the left side of Fig. 4. The ARTCC decision support tool uses surface trajectory-based OFF time predictions for departure

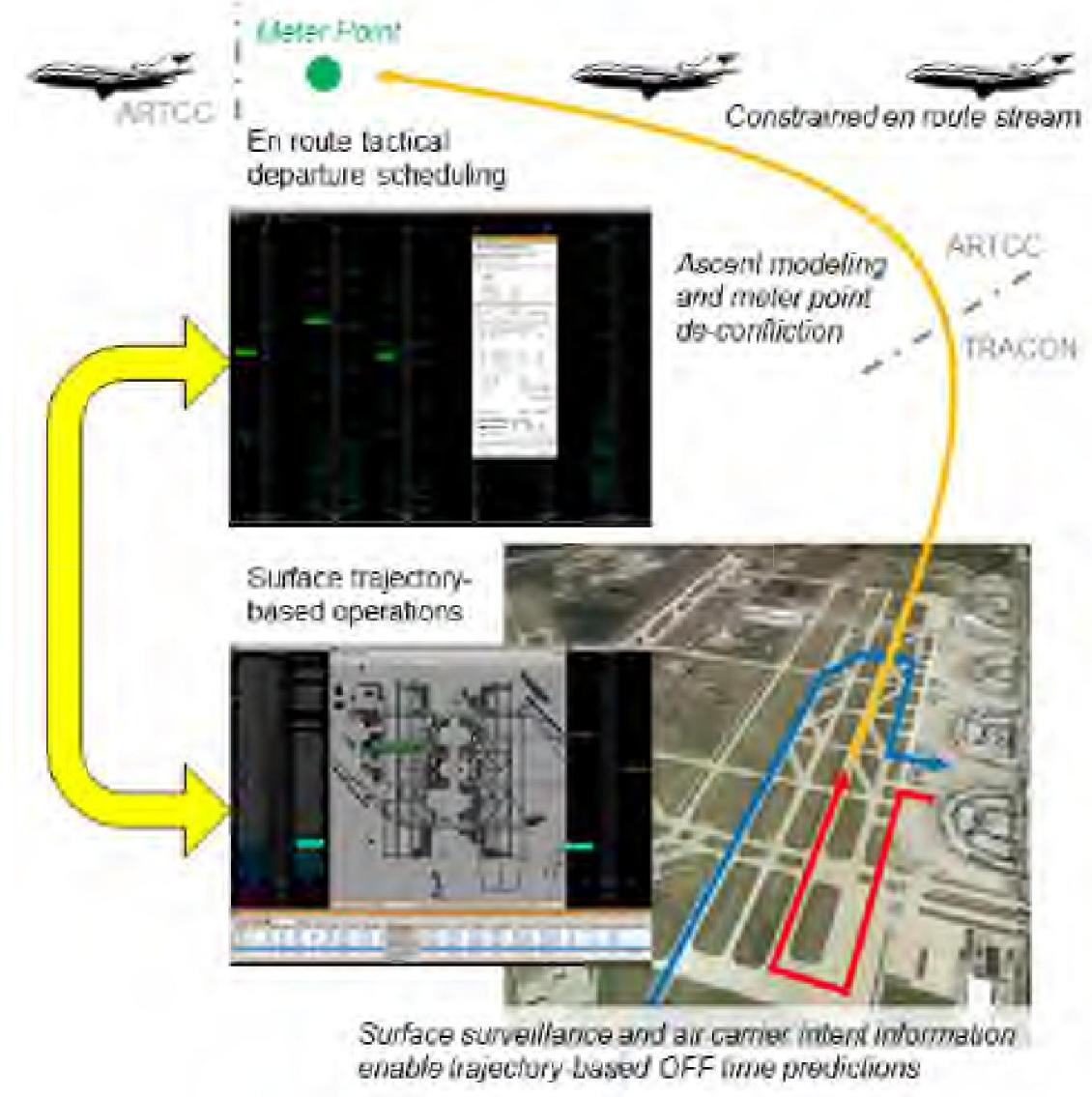

Figure 4. PDRC concept overview diagram.

scheduling and coordinates release times with the ATCT surface trajectory-based decision support tool. The ATCT tool predicts OFF times for use by the ARTCC tool in tactical departure scheduling and coordinates release times with the ARTCC decision tool.

The focal point for PDRC is the OFF point in Fig. 4 where the red trajectory joins with the gold trajectory on the departure runway. The ATCT decision support tool computes surface trajectories to this point to develop OFF time estimates. The ARTCC decision support tool computes ascent trajectories from this point to the merge point in the overhead stream for tactical departure scheduling. 


\section{System Design}

This section describes the PDRC software components and provides a walk-though of the nominal scenario for the PDRC operational concept.

\section{A. Prototype Software}

The PDRC research activity strives to be implementation neutral, meaning that the research results will be generally applicable regardless of which specific surface or en-route tools are used. Generally applicable research results will include the overall operational concept, OFF time prediction accuracy requirements, and information exchange requirements. The prototype software used for development and evaluation may not reflect eventual implementation systems.

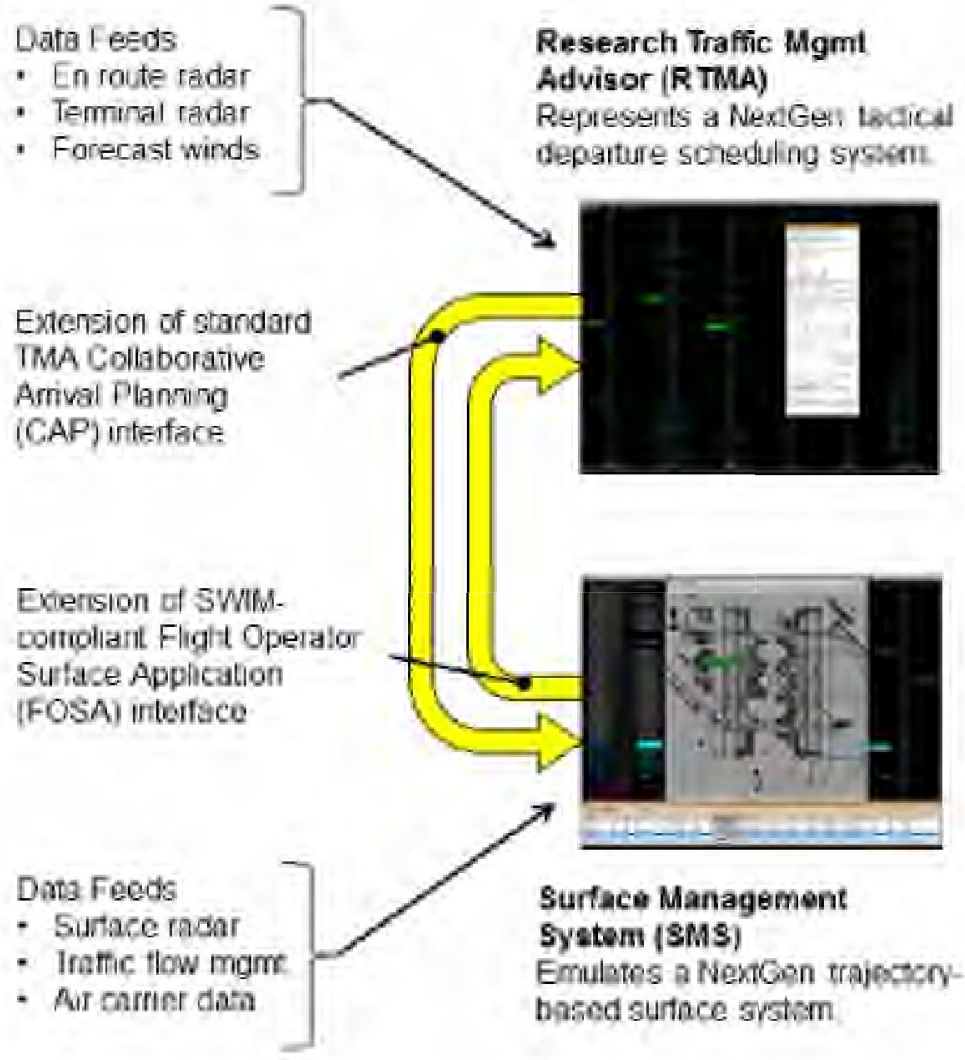

Figure 5. PDRC prototype software overview diagram.
Figure 5 provides a high-level overview of the prototype software being used for PDRC. The upper portion of this figure shows the ARTCC tactical departure scheduling decision support tool. The PDRC prototype utilizes Research Traffic Management Advisor (RTMA) for this component. The lower portion of this figure shows the ATCT decision support tool. The PDRC prototype uses the Surface Management System (SMS) for this component. The RTMA and SMS decision support tools and PDRC two-way interface are described below

\section{Research TMA (RTMA)}

RTMA was derived from a recent release (3.10.0) of the FAA's operational TMA software. RTMA has been modified to build and execute in NASA's research environment (i.e. Linux operating system on Intel processors) and configured to run without the elaborate monitor \& control system that supports the FAA's operational TMA installation.

PDRC provided the original motivation for creating RTMA; however, this research tool promptly found other applications. NASA and the FAA are both using RTMA to support various R\&D efforts and the agencies are actively collaborating to further develop RTMA capabilities. The immediate next step is to upgrade RTMA to match the current operational TMA version which is 3.12.0.

The RTMA development strategy maximizes commonality with the FAA operational TMA baseline to facilitate technology transfer. RTMA includes a new Surface Data Interface (SDIF) module that enables communication with SMS via the Flight Operator Surface Application (FOSA) interface. RTMA also includes scheduling algorithm (i.e. Dynamic Planner and Meter Point Dynamic Planner) and user interface modifications to enable use of SMS trajectory-based OFF time predictions. RTMA retains TMA's two trajectory-based tactical departure scheduling functions: TMA EDC for the outbound situation and TMA arrival metering "internal" departure scheduling for the inbound situation.

The upper left portion of Fig. 5 depicts the data feeds required by RTMA which are the same as for operational TMA systems. Flight plans and tracks are provided by surveillance data feeds from ARTCC (home and all adjacent facilities) Host computers. Surveillance data from the ARTS or STARS computers at all involved TRACON facilities is also required. Finally, the system uses Rapid Update Cycle (RUC) forecast winds aloft information. 


\section{Surface Management System (SMS)}

In 2003 NASA successfully demonstrated a prototype Surface Management System (SMS) decision support tool at Memphis. ${ }^{13}$ NASA transferred the SMS technology to the FAA which developed it into the Surface Decision Support System (SDSS) research platform. The FAA's Advanced Technology Development \& Prototyping Group uses SDSS at various FAA test beds to support Surface Trajectory-Based Operations (STBO) research activities. ${ }^{14}$

SMS continues to be NASA's primary platform for NextGen surface automation research. SMS supports various NASA research activities ${ }^{15}$ in addition to the PDRC research activity described in this paper. These NASA research activities contribute to a common SMS software baseline. Active software development collaboration between NASA and the FAA STBO project has resulted in a high degree of commonality between SMS and SDSS - to the point where the SMS and SDSS names are often used interchangeably.

SMS is a full-featured decision support tool designed to help controllers, traffic managers, and air carriers manage the movements of aircraft on the surface of busy airports. SMS includes traffic management functions for use in ATCTs, TRACONs, and ARTCCs. PDRC utilizes SMS traffic management functions associated with the CFR procedure as well as the underlying surface trajectory computations that support all SMS functions. SMS produces trajectory-based OFF time predictions by combining air carrier intent information with surface surveillance data and departure queue projections.

The lower left portion of Fig. 5 depicts the data feeds required by SMS. At NTX the surface surveillance data comes from the DFW Airport Surface Detection Equipment, Model X (ASDE-X) system. Air carrier data (i.e. gate assignments, equipment assignments and arrival \& departure time estimates) were obtained from the DFW Airport Operations Database (AODB) system. RTMA is the source for flight plans and airborne surveillance data as described below.

\section{PDRC two-way interface}

PDRC is primarily a systems integration research activity where two relatively mature decision support tools (RTMA and SMS) have been combined to create a new capability. PDRC technology enables RTMA and SMS to share information that reduces uncertainty in the tactical departure scheduling process. The new PDRC technology includes a two-way communications interface between RTMA and SMS represented by the double yellow arrows on the left side of the Fig. 5.

The PDRC two-way interface leverages existing SMS and TMA communications capabilities. The RTMA-toSMS interface uses the existing SDSS interface to the TMA Collaborative Arrival Planning (CAP) data feed. The CAP message set was extended to include PDRC scheduling information. The new SMS-to-RTMA interface is an extension of SDSS' SWIM-compliant FOSA interface.

A primary function of this interface is to deliver the SMS OFF time predictions to RTMA. Specifically, these are the Undelayed and Predicted Coordinated OFF Times (UCOT and PCOT). UCOT is the earliest time that SMS predicts that the flight could takeoff if there was no congestion on the airport surface. The PCOT is the time that SMS projects that the flight would takeoff based on its scheduling of aircraft actions under the active scheduling constraints. PCOT is used by RTMA in tactical departure schedule calculations. UCOT can be used to project the congestion-induced ground delay a flight will experience. The PDRC research team may explore the use of UCOT to provide "credit" for ground delay when establishing the tactical departure schedule.

The PDRC two-way interface also enabled a novel solution to a flight plan and airborne surveillance data source requirement. Typically SMS and SDSS use Traffic Flow Management System (TFMS) data as the source for flight plans and airborne surveillance data. PDRC requires the flight data in the component decision support tools (i.e. RTMA and SMS) to correspond as closely as possible. This requirement was met by using RTMA's existing CAP interface to deliver ARTCC and TRACON flight plan and track data to SMS. This approach follows the example of earlier NASA work, ${ }^{16}$ providing SMS with new, high-quality surveillance data sources and ensuring that both PDRC components are operating on identical sets of flight information.

\section{B. Operational concept scenario}

The CFR procedure is a collaborative effort between the ARTCC traffic management unit responsible for meeting the overhead stream constraints and the ATCT controllers responsible for releasing departing aircraft. The PDRC concept automates portions of this collaborative departure scheduling effort. Specifically, PDRC's SMS component automatically predicts the earliest achievable OFF times for all departure flights. For flights subject to CFR restrictions the SMS OFF time predictions are automatically communicated to RTMA for use in tactical departure scheduling functions. The PDRC two-way interface also facilitates inter-facility coordination required to establish the agreed upon release time. 
Although no nationwide guidance exists, inter-facility agreements generally call for CFR flights to depart within a three-minute window. The CFR three-minute compliance window is often structured to allow departure two minutes prior to or one minute later than the target coordinated departure time. The compliance window is biased because it is easier to slow an early departure to fit into a slot than it is to accelerate a late departure. Tactical CFR precision requirements are significantly higher than for strategic TMIs. ${ }^{9}$ Even so, the three-minute window seems rather imprecise when one considers that this equates to a 21 -to- 24 mile slot at the en route meter point.

It should be noted that application of PDRC automation does not fundamentally alter the CFR procedure. ARTCC and ATCT roles and responsibilities may remain the same and CFR operations may be conducted per existing inter-facility Letters of Agreement. TMA is widely used for tactical departure scheduling to meet CFR restrictions, and TMC interactions with the TMA departure scheduling functions are essentially unchanged with the PDRC system. Thus, only the SMS-related portions of the following scenario represent material changes to the present-day CFR procedure.

Figure 6 shows annotated portions of the SMS and RTMA Graphical User Interfaces (GUIs). These images will be used to help illustrate key steps in the nominal PDRC operational scenario. The left side of Fig. 6 shows a portion of the SMS GUI as it would be configured for use during a CFR procedure. The image shows portions of the surface traffic map display, the CFR timeline, and the flight table at the bottom. The right side of Fig. 6 shows a small portion of the RTMA timeline GUI. This particular example is for an outbound tactical departure scheduling situation, so the GUI is setup as the ZFW EDC system. The timeline shown is for the TVN meter arc which is situated very near the TORNN arc shown in Fig. 2. TMA's departure scheduling dialogue box is also shown in the image.

The two GUI images in Fig. 6 are from the same CFR scheduling event. Flight AAL1560 from DFW to IAH is subject to CFR restrictions. Surface surveillance is active for the flight which is shown northbound on taxiway G near the left side of Fig. 6. The RTMA GUI image was captured about 20 seconds prior to the SMS GUI image. The RTMA GUI image shows the result of using SMS OFF time predictions for outbound tactical departure scheduling on AAL1560. The SMS GUI image shows the release time being accepted.

The PDRC operational concept scenario will now be described with reference to Fig. 6 . The actors in this operational concept scenario are (nominally) both Traffic Management Coordinators (TMCs). One TMC is located

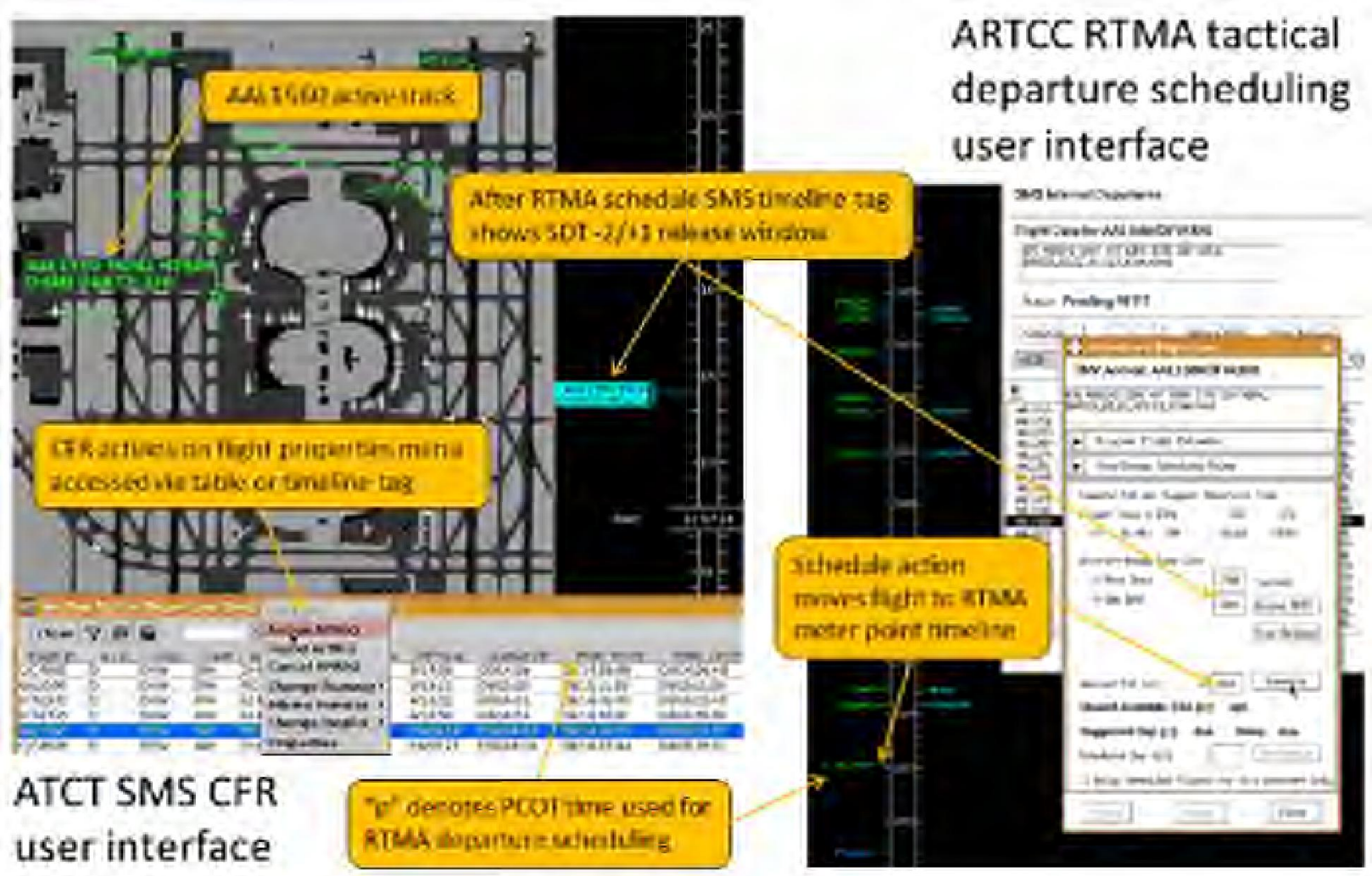

Figure 6. ARTCC and ATCT interactions for the nominal scenario in the PDRC operational concept. 
in the ARTCC Traffic Management Unit (TMU) and the other is located in the ATCT. For simplicity these TMCs are identified as Center and Tower in the following discussion.

\section{CFR restriction notification}

The CFR notification process varies by facility. At ZFW, the Center notifies D10 TRACON which, in turn, notifies the Tower(s) that CFR restrictions have been implemented for a given destination and time range. Notifications are via inter-facility voice calls. After the initial notification via D10 TRACON the Tower communicates directly with the Center when making CFR requests. Both ZFW and D10 also enter the CFR restrictions in their electronic logging systems.

\section{CFR activation in SMS}

In response to receiving a CFR restriction, the Tower will use the SMS "Add/Remove APREQ Schedule" dialog to enter a new CFR. The CFR will include the destination airport or jet route and the time range that are used to determine which flights are subject to the CFR restriction. Flights subject to the CFR restriction are entered into a list used by RTMA and SMS to track which flights are actively being coordinated.

\section{Request for Release Time (RFRT)}

As a flight becomes ready for departure, the Tower selects "Request Departure Time" from the SMS "Flight Properties" menu (accessed for the specific flight from the timeline or flights table). This causes SMS to send a Request For Release Time (RFRT) to RTMA. The request will include time projections for both UCOT and PCOT times. The flight's PDRC status is updated to "PENDING RFRT" and displayed on both systems (SMS and RTMA).

\section{Response to the Request for Release Time (RFRT)}

The Center will respond to RFRT requests using RTMA's "Schedule a Departure" dialog which has been enhanced to display SMS scheduling information. The Center will select one of the following actions;

- FREE RELEASE, which tells the Tower that the flight may be released at their discretion.

- SCHEDULE USING SMS, which causes RTMA to schedule the flight for departure at the earliest time that is equal to, or later than, PCOT. If the Center FREEZES or ACCEPTS the Suggested Departure Time via the dialog or manually schedules via timeline actions, then RTMA forwards the Scheduled Departure Time (SDT) to SMS. At that point, the flight will be assigned a PDRC status of "SDT SENT".

- DELAY a release decision until later. If the delay is more than a short period of time, this action will be supplemented with direct communications with the Tower for coordination.

The RTMA GUI image on the right side of Fig. 6 was captured just after the Center clicked the "Schedule" button followed by the "Freeze" button. Flight AAL1560 is now shown as scheduled and frozen on the TNV meter arc timeline. The SDT of 18:06 has been returned to SMS and is reflected by the location of Flight AAL1650 on the SMS runway timeline.

5. Response to Scheduled Departure Time (SDT)

When an SDT is sent to SMS, the Tower determines if the RTMA supplied SDT is acceptable and will select one of the following actions;

- SDT is acceptable, which will prompt notification to the Center and an update of the flight's PDRC status to "SDT ACCEPTED." On the SMS display, this will cause the timeline data block text to include "APRQ:mm/mm", where the first "mm" shows the beginning of the departure release window and the second "mm" shows the end of the window. The data block highlighting will indicate whether the flight's current predicted OFF Time is before, after, or within a configurable tolerance of the SDT.

- SDT is not acceptable, which will prompt notification to the Center and an update of the flight's PDRC status to "SDT REJECTED". In this case, the Tower and Center must resolve the situation via voice communications. After this Tower/Center coordination, the Center may reschedule the flight, unschedule the flight, or remove the flight from active SMS/RTMA coordination.

The SMS GUI image on the left side of Fig. 6 was captured just as Tower used the "Flight Properties" menu to select the "Accept APREQ" action. The AAAL1560 data tag is aligned with 18:04 on the SMS timeline and shows 04/07 reflecting the $-2 /+1$ minute compliance window applied to the 18:06 release time that the Tower is agreeing to meet. 


\section{Departure release management}

Tower traffic control tactics for CFR flights are determined by the Center's response to the RFRT:

- If a Free Release has been sent from RTMA to SMS, then the flight's timeline tag reverts to normal text display and "APRQ:FREE" is displayed in the tag. The Tower can then manage that flight's departure as if no CFR restriction were in place.

- If a SDT has been provided and accepted, then the Tower performs tasks to meet the RTMA SDT. These may include: flight strip marking and manipulation procedures; ATC control of aircraft surface movements, arrivals, and departures; and monitoring SMS's continuously updated projections of departure time estimates.

\section{Field Test}

This section begins with an overview of the July 2011 initial field test. The overview is followed by descriptions of the test apparatus and environment. Initial field test operations are then described along with a report of observations.

\section{A. Overview}

The initial PDRC field test began on 13 July 2011 and ended on 29 July 2011. PDRC user interfaces and NASA observers were stationed at four positions: the ZFW ARTCC TMU, the DFW East ATCT, the American Airlines (AA) DFW ramp tower, and the NTX laboratory. This test included 30 trained TMCs and Supervisor TMCs (STMCs) at ZFW ARTCC, and the DFW ATCT. Locations of the observation positions are shown in Fig. 7, and described in detail in a later section. Figure 7 shows a planview image of the NTX environment. DFW airport is to the north with runways highlighted in yellow. ZFW and the NTX laboratory are located about 3 miles southwest of the airport. Field observation locations and systems are shown in the inset images.

There were three objectives for this initial PDRC field test: 1) test apparatus checkout; 2) TMC training; and 3) live-traffic, operational-environment, TMC shadow evaluations. The test plan included provisions for conducting initial operational evaluations upon completion of the primary objectives. The first week was devoted to test apparatus checkout and controller training. During the second week, PDRC was used in a shadow mode that

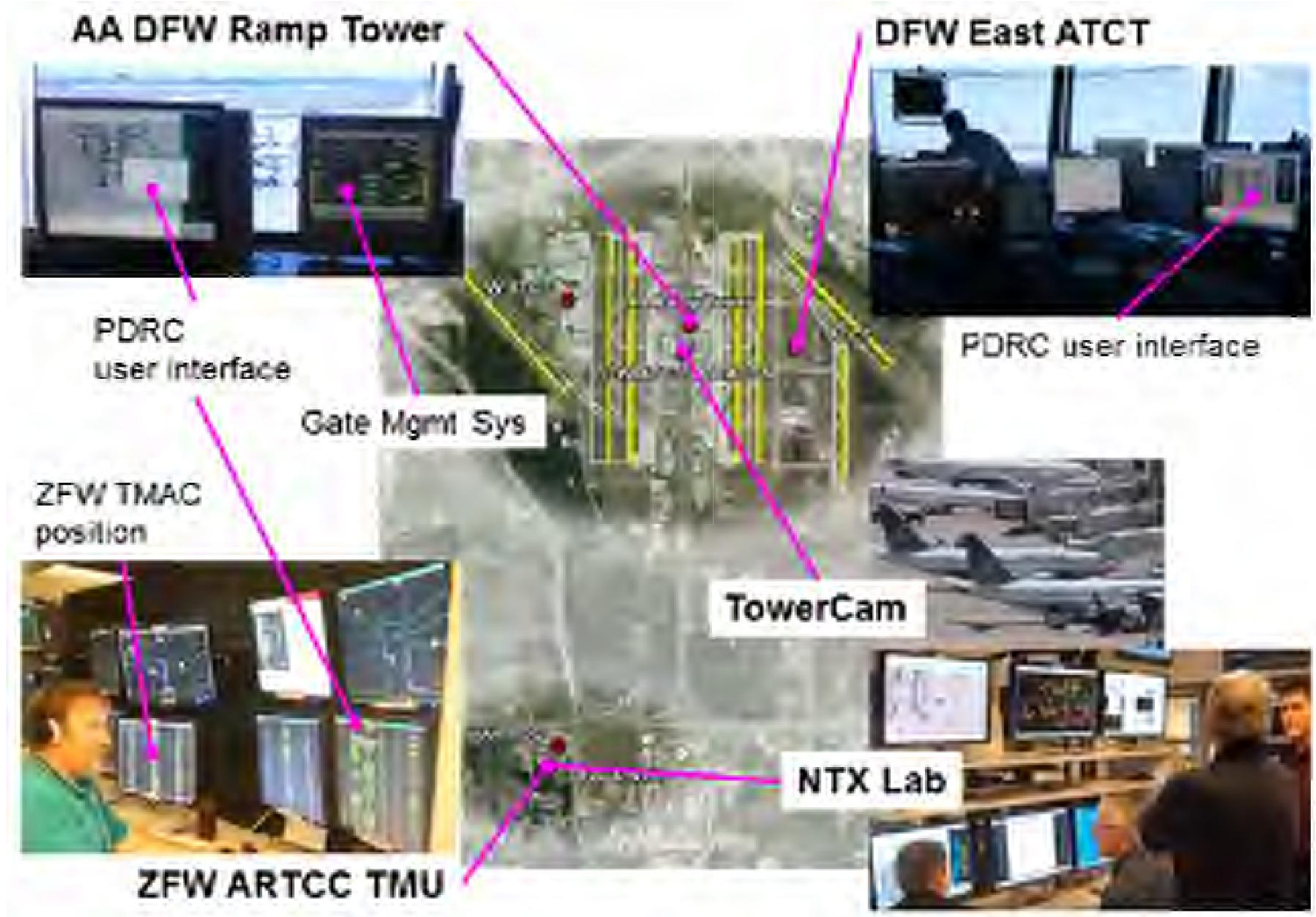

Figure 7. PDRC field test observation positions in the vicinity of DFW airport.

10

American Institute of Aeronautics and Astronautics 
evaluated the advisories without affecting actual operations. The 12-day test period concluded with several operational evaluation runs where TMCs successfully used PDRC advisories to schedule actual DFW departures.

\section{B. Apparatus}

The primary apparatus for the July 2011 field test was the PDRC system itself. As described in Section III(A), a PDRC system consists of:

- $\quad$ RTMA decision support tool including GUI clients (typically 3 per system)

- $\quad$ SMS decision support tool including GUI clients (typically 4 per system)

- Live data feeds for RTMA and SMS including ARTCC, TRACON, and ASDE-X surveillance, RUC forecast winds aloft, and air carrier data

- PDRC two-way interface connecting RTMA and SMS

Two separate PDRC systems were required for this test. One PDRC system had RTMA configured to match the operational ZFW TMA EDC system to support evaluation of the outbound tactical departure scheduling situation. The other PDRC system had RTMA configured to match the operational ZHU TMA arrival metering system to support evaluation of the inbound tactical departure scheduling situation.

Each of the PDRC systems was hosted on its own dedicated Linux server configured with two $3.0 \mathrm{GHz}$ dual-core CPUs and 8GB RAM. Each PDRC server ran all of the RTMA and SMS computational processes, the associated GUI clients, and services for distributing the GUI clients to remote user displays.

The PDRC field test depended upon a number of NTX-built research support systems. A remote framebufferbased graphical desktop sharing system was used to distribute the PDRC GUI clients to the various end-user display machines. This desktop sharing system included digital video recording of all displays for post-test review. A Voice over Internet Protocol (VoIP)-based audio conferencing system enabled research observers to freely communicate with other members of the NASA team while minimizing interference with the operational environments. All research team communications were digitally recorded to capture research observer comments and observations for post-test review. Finally, various purpose-built systems were used to automatically record and analyze data from the RTMA, SMS and operational TMA systems.

The NTX-built TowerCam video surveillance system deserves special mention due to its central role in the PDRC field test. TowerCam consists of two remote control pan/tilt/zoom cameras mounted in the DFW Center ATCT. TowerCam enables NASA research observers to visually monitor traffic in nearly all of the DFW airport movement areas and some of the ramp and gate areas. For this field test, TowerCam was particularly important for monitoring pre-pushback events for non-American Airlines flights operating from Terminal E.

FAA-provided TFMS and TMA displays in the NTX lab were immensely valuable. TMA repeater displays for ZFW and ZHU enabled NASA researchers to verify that the PDRC RTMA systems were properly configured and performing correctly. The TFMS Traffic Situation Display (TSD) provided situational awareness, and the NTML system delivered real-time information on CFR restrictions. The latter was useful for pre-test planning sessions and to supplement reports from the ARTCC and ATCT observers.

\section{Environment}

NASA researchers were stationed at four different positions during the PDRC field test. These observation positions are illustrated in Fig. 7 and described below.

\section{ZFW ARTCC TMU}

The ARTCC portion of the PDRC prototype system is operated from this position. This corresponds to the Center role in the PDRC operational concept scenario described in Section III(B) above. This position is shown in the lower left portion of Fig. 7. The four PDRC displays shown in the figure are immediately adjacent to operational TMA displays used by the ZFW Traffic Management Arrival Coordinator (TMAC). Additional operational TMA displays are located about 10 feet away at the ZFW Traffic Management En Route Coordinator (TMEC) position.

The ZFW TMU is relatively self-contained and staffed by TMCs and STMCs. These were all trained on the PDRC decision support tools, and most of them participated in the shadow and operational evaluations.

The NASA observer at this position was responsible for supporting the TMC in the use of PDRC and collecting observational data. These observations included: TMC comments, TMC interactions with PDRC, record of flights affected by PDRC, entry of PDRC times into the operational TMA systems, characterization of the overhead stream flow and any negative impacts to this, notes on required vectoring or speed control, slot acceptability, etc. This observer monitored the current RTMA configuration to ensure that it matched the operational TMA configuration. This observation position also provided the test director with valuable situational awareness regarding the current ZFW TMU environment. 


\section{DFW East ATCT}

The ATCT portion of the PDRC prototype system was operated from this position. This corresponds to the Tower role in the PDRC operational concept scenario described in Section III(B) above. This position is shown in the upper right portion of Fig. 7. The single PDRC display shown in the figure is located on the right side of the TMC desk.

The TMU functions in the ATCT are much less segregated than they are in the ARTCC. Consequently, all ATCT personnel were involved in the PDRC field test, some directly and others indirectly. The direct involvement was managed by ATCT TMCs who are part of the Metroplex Traffic Management Unit that also contains STMCs and TMCs from DFW TRACON.

The NASA observer at this position was responsible for supporting the TMC in the use of PDRC and collecting observational data. These observations included: TMC comments, TMC interactions with PDRC, record of flights affected by PDRC, and notable surface events (e.g. long departure queue, equipment malfunction, configuration change, etc.). This observer monitored the current SMS configuration and ensured that aircraft runway assignments were correct. This observation position also provided the test director with valuable situational awareness regarding the current DFW ATCT environment.

\section{AA DFW Ramp Tower}

This PDRC field observation position was established to gain a better understanding of the available air carrier data and to experiment with it through manual entry into the PDRC system. As described in Section III(A), the SMS component of PDRC is currently using an air carrier data source (i.e. DFW Airport AODB) primarily intended for public consumption (e.g. airport terminal flight information display systems). NASA is investigating the potential benefit of obtaining higher-quality data via direct air carrier participation. This interest is motivated, in part, by earlier research ${ }^{17}$ demonstrating the use of air carrier pre-departure event information to improve OUT predictions.

This position is shown in the top left corner of Fig. 7. The NASA observer at this position used American Airlines' Gate Management System (GMS) to obtain the best available, real-time data regarding gate assignments and OUT (i.e. pushback) time estimates. The NASA observer manually updated SMS as required to reflect the information in GMS. This observer had a secondary assignment of collecting actual observed OUT times for a sample of flights. Comparison of these observed actual OUT times with estimated OUT times and actual OUT times reported by other sources (e.g. Aviation System Performance Monitoring) is expected to provide valuable insights. Finally, this observer was in a unique position to gather contextual information from the air carrier perspective.

\section{NTX Laboratory}

The test director and test engineer were stationed in the NTX Laboratory shown in the lower right corner of Fig. 7. The test director was responsible for the overall conduct of the field test and monitored PDRC systems and position observers from the laboratory. A primary consideration for the test director was the status of the various operational environments (i.e. ZFW TMU, DFW ATCT, and AA ramp tower) where PDRC equipment and observers were stationed. The test director coordinated frequently with operational managers regarding the test. Real-time reports from NASA observers provided the test director with valuable situational awareness.

The test engineer used an array of tools in the NTX laboratory to monitor the configuration and performance of all PDRC systems, monitor FAA systems (i.e. TFMS and TMA repeater) to enhance research team situational awareness, and monitor data recording systems. The test engineer also maintained redundant observational logs and served as the primary contact for observers at the remote positions.

\section{Operations}

Table 1 documents the dates and times during which test operations were conducted. NASA observers were on position approximately 61 hours during the 12 test days.

The first week was devoted to the system checkout and TMC training objectives. The TMC training can be characterized as on-the-job training. NASA observers would familiarize TMCs with PDRC decision support tool functions and answer any questions that arose. This on-the-job training was conducted in parallel with the NASA system checkout runs.

The second week focused on live-traffic shadow evaluations. The demarcation between TMC training and TMC shadow evaluations proved to be fairly subtle. As TMCs became familiar with the system, they naturally began to provide feedback on PDRC system performance and the usability of the PDRC-generated tactical departure schedules. 
The shadow evaluation period also included significant coordination with TMCs and STMCs concerning procedures for conducting PDRC operational evaluations. This was more of a challenge at the ARTCC than at the ATCT. The field test setup at the ATCT closely approximates the envisioned end-state for PDRC. The ARTCC environment was more artificial due to the requirement to run RTMA in parallel with the operational TMA and manually transfer scheduling inputs from one system to the other.

By 27 July 2011 the NASA team and the ARTCC and ATCT TMCs and STMCs deemed the PDRC system ready for operational evaluations. However, an unusually persistent high-pressure weather system over much of the country significantly reduced the instances of tactical departure scheduling (i.e. CFR procedure use) during the test period. This was not a major factor during the system checkout and training periods, but it did impact the number of opportunities for meaningful shadow and operational evaluations at the end of the test period. Tropical storm Don in the Gulf of Mexico, promised some CFR activity during the last two days of the test; however, this proved to be too much of a good thing on the final day as Don forced a ground stop for DFW traffic to IAH and HOU.

The research team was able to collect data on five operational evaluation runs where controllers successfully used PDRC advisories to schedule actual DFW departures. These operational runs were all for the outbound tactical departure scheduling situation using EDC. Qualitative TMC feedback from these initial operational evaluations indicated that PDRC takeoff time estimates and en route departure schedules were usable and show promise of providing the expected benefits.

\section{E. Observations}

This section documents notable observations and lessons learned during the July 2011 PDRC field test. These observations were gathered during both the shadow and operational evaluation phases of the test.

\section{SMS OFF time predictions - qualitative assessments}

The PDRC research team systematically collected both quantitative and qualitative data on SMS OFF time predictions. The qualitative data was gathered by the NASA observer stationed in the DFW East ATCT. For each flight subject to a CFR procedure, the NASA observer would ask the TMC to provide feedback on the acceptability of the OFF time estimate in real-time as SMS was making the prediction. Overall, TMC feedback on SMS OFF time predictions was positive with the large majority of comments assessing the predictions as a "good" or "acceptable." The TMC's indicated that they would be comfortable using the SMS predictions for CFR purposes.

There were cases where the TMC did not agree with the SMS OFF time prediction. In nearly all of these instances, the TMC deemed the SMS taxi time estimate to be too high. Many of these cases were associated with a problem in the SMS modeling logic which allowed flights actively taxiing in the air movement area to be sequenced behind inactive flights. A simple change to the modeling logic was implemented prior to the final week of the test. This change appeared to significantly improve SMS sequencing of active vs. inactive flights; however, additional analysis is required to identify the best long-term solution.

In at least one instance, the TMC assessed the SMS taxi time estimate as "too high" but later decided that the prediction was "good." In this case, the observer noted that high-than-normal departure queue congestion made early manual prediction difficult.

\section{Imprecision in present-day CFR procedures}

As noted in Section III(B), the CFR departure release time compliance window varies somewhat by facility. ZFW and DFW use the typical $-2 /+1$ window. During the July 2011 field test, the PDRC research team found that the CFR compliance situation is further complicated by varying interpretations and practices regarding the actual target release time within the three-minute window. Existing procedures do not specify a precise target within this 
window, so ATCT controllers and TMCs are free to use the entire window as they manage the flow of traffic on the runways. Also, the CFR communication between ARTCC and ATCT frequently involves the exchange of a single time value. The meaning of this single time value may be subject to different interpretations, and it requires TMCs to mentally calculate the corresponding compliance window. The PDRC research team is currently working with ZFW and DFW TMCs to explore options for increasing the precision of CFR communications during future PDRC operational evaluations. Previous research ${ }^{6,7}$ has demonstrated the value of automation-assisted communication of departure release times. Thus, the solution will likely involve more explicit use of the PDRC-generated release times in inter-facility CFR communications.

\section{The challenge of prototype system artifacts}

Researchers must often account for artificial elements introduced into an experiment by the very nature of the research environment. For PDRC, the principal research artifact was the requirement to manually transfer scheduling information from the research TMA system to the operational TMA system. The PDRC concept of operations calls for an en route tactical departure scheduling system (e.g. TMA) to coordinate CFR times with a trajectory-based surface decision support tool (e.g. SMS). For this initial field test, the PDRC prototype had to run in parallel with the operational TMA system. Thus, tactical departure scheduling information from PDRC had to be manually transferred to the operational system. A significant portion of the shadow evaluation period was devoted to refining this manual transfer procedure. Even after refinement, this manual data transfer process proved somewhat cumbersome. The PDRC research team is currently working with ZFW TMCs to improve this process for future operational evaluations.

\section{Gate-to-spot prediction uncertainty}

SMS trajectory-based OFF time predictions are highly dependent on surface surveillance data. Absent aircraft state information from surveillance data, trajectories must be manufactured based upon parking gate location and OUT (i.e. pushback) time estimates. The uncertain nature of airport ramp or apron operations is widely recognized as an impediment to long-range prediction of OFF times. Consequently, the July 2011 PDRC field test included, as a secondary objective, the collection of airport ramp event data from a variety of perspectives: 1) SMS predicted and "actual" OUT times, 2) AA gate management system predicted and "actual" OUT times, 3) DFW gate docking system OUT times, 4) visual observations of actual OUT times, 5) identification of first track events, and 6) contextual observations that help explain why some of the uncertainty occurs.

Detailed analysis of these gate-to-spot observations is currently underway, and much work remains to be done. However, some preliminary results can be reported from the field test notes. Observations confirmed earlier, informal reports of wide variation in aircraft transponder activations. Transponder activations were observed during virtually every segment of the gate-to-spot movement. In almost every case, transponders were activated prior to reaching the spot - but usually a short distance before the spot. In very few cases were transponders activated right at pushback. In several cases, the transponder appeared to deactivate shortly after activating. It is hypothesized that these on/off/on events are associated with the transition from APU to engine electrical power.

These preliminary results indicate that the uncertainty of pre-spot predictions may be too high for use in tactical departure scheduling. Also, it seems likely that improvements in gate-to-spot estimates will be driven by data sources other than transponder-dependent ASDE-X. For example, OUT event detection and reporting systems (e.g. ACARS, or gate dock systems) can provide reliable notification of pushback events that may be used to significantly improve gate-to-spot predictions.

\section{Target-of-opportunity evaluation methodology}

The July 2011 field test experience reinforces the need to develop an agile and opportunistic evaluation methodology both for PDRC and tactical departure scheduling in general. As noted above, the weather was uncooperative with a persistent high-pressure system over much of the country significantly reducing the instances of tactical departure scheduling (i.e. CFR use). Outbound tactical departure scheduling was much lower than normal, and inbound tactical departure scheduling associated with ZHU metering to IAH was extraordinarily low. Fortunately, this did not significantly impact this initial field test with its focus on system checkout, training, and shadow evaluations. However, future tests will emphasize operational evaluation data collection. The PDRC research team is working to adapt the test systems and procedures to support target-of-opportunity operational evaluations and improve data collection efficiency. 


\section{Conclusion}

This paper presents an overview of tactical departure scheduling along with definitions for and examples of the inbound and outbound tactical departure scheduling situations. A companion paper ${ }^{9}$ provides an in-depth examination of tactical departure scheduling, and identifies shortfalls in present-day tactical departure scheduling operations. These shortfalls may be eliminated by reducing uncertainty in OFF time predictions.

The PDRC concept was developed to address the tactical departure scheduling shortfall associated with uncertain OFF time predictions. The PDRC concept calls for the use of surface trajectory-based takeoff (OFF) time predictions for en route tactical departure scheduling during CFR procedures.

A PDRC prototype has been assembled by linking a research version of TMA (RTMA) to the SMS research platform. TMA was selected for the PDRC prototype because it includes functions for both outbound and inbound en route tactical departure scheduling, and these TMA functions are widely used in the NAS. SMS is representative of a NextGen decision support tool for the ATCT environment and provides the trajectory-based OFF time predictions and CFR procedure functions required for PDRC development and evaluation.

An initial field test of the PDRC prototype was conducted during July 2011 at the NTX Research Station in Dallas/Fort Worth. The field test accomplished its three primary objectives which were system checkout, TMC training, and live-traffic, operational-environment TMC shadow evaluations. The test concluded with initial operational evaluation runs where TMCs used PDRC-generated tactical departure schedules to meet CFR constraints for five actual DFW departures. TMC feedback obtained from this initial test was immensely valuable and is being used to refine plans for future operational evaluations.

This paper presented initial field test results in the form of qualitative observations. Quantitative results are not yet complete, but they will include an analysis of OFF time prediction performance and an assessment of en route scheduling performance with particular attention to slot utilization and controller workload. Results from this initial field evaluation, along with findings from associated analytical studies, ${ }^{9,18}$ will be used to refine the PDRC concept and decision support tools, and to improve the methodology for planned follow-on operational evaluations.

\section{Acknowledgments}

This work was supported by the NASA NextGen Systems Analysis, Integration and Evaluation (SAIE) Project. The authors would like to acknowledge the substantial support provided by FAA personnel at the Fort Worth ARTCC Traffic Management Unit, Dallas/Fort Worth ATCT, and Metroplex Traffic Management Unit. We would also like to thank the management and staff at American Airlines DFW Ramp Tower for their kind hospitality. Finally, we wish to thank our outstanding colleagues and friends at NTX who were essential to the success of the PDRC field test.

\section{References}

${ }^{1}$ JPDO, “Integrated Work Plan for the Next Generation Air Transportation System," version FY13, http://jpe.jpdo.gov/ee/request/home.

${ }^{2}$ FAA, "NextGen Mid-Term Concept of Operations for the National Airspace System,” version 2.1, September, 2010.

${ }^{3}$ Borowski, M., Hu, S., Jones, R., and Levin, K., "Integrated Arrival, Departure, and Surface (IADS) Concept for the MidTerm,” MITRE CAASD MTR100348, September, 2010.

${ }^{4}$ Audenaerd, L., Burr, C., and Morgan, C., "Surface Trajectory-Based Operations (STBO) Mid-Term Concept of Operations Overview and Scenarios,” MITRE CAASD MP090230, September, 2009.

${ }^{5}$ Nene, V., and Morgan, C., "A Mid-Term Concept of Operations for a Tower Flight Data Manager (TFDM)," MITRE CAASD MP090169, September, 2009.

${ }^{6}$ Kopardekar, P., Green, S., Brinkman, C., Thompson, P., Evans, M., and Davis, D., "Making Internal Departure Release Operations More Efficient," AIAA 2004-6346, AIAA 4th Aircraft Technology, Integration, and Operations (ATIO) Forum, Chicago, Illinois, 20-22 September, 2004.

${ }^{7}$ Kopardekar, P., Green, S., and Thompson, P., "Improving Efficiency of Departure Release Communications for En Route Overhead Traffic Flow Management," 23 ${ }^{\text {rd }}$ Digital Avionics Systems Conference, Salt Lake City, Utah, 24-28 October, 2004.

${ }^{8}$ Doble, N., Timmerman, J., Carniol, T., Klopfenstein, M., Tanino, M., and Sud, V., "Linking Traffic Management to the Airport Surface: Departure Flow Management and Beyond," Eighth USA/Europe Air Traffic Management Research and Development Seminar (ATM2009), Napa, California, 29 June - 2 July, 2009.

${ }^{9}$ Capps, A., Engelland, S, "Characterization of Tactical Departure Scheduling in the National Airspace System," AIAA $11^{\text {th }}$ Aviation Technology, Integration, and Operations (ATIO) Conference, Virginia Beach, Virginia, 20-22 September, 2011 (submitted for publication).

${ }^{10}$ Swenson, H. N., Hoang, T., Engelland, S., Vincent, D., Sanders, T., Sanford, B., and Heere, K., "Design and Operational Evaluation of the Traffic Management Advisor at the Ft. Worth Air Route Traffic Control Center," First USA/Europe Air Traffic Management R\&D Seminar, Saclay, France, June, 1997. 
${ }^{11}$ Farley, T. C., Landry, S. J., Hoang, T., Nickelson, M., Levin, K. M., Rowe, D., and Welch, J. D., "Multi-Center Traffic Management Advisor: Operational Test Results," AIAA-2005-7300, Proceedings of the 5th AIAA Aviation Technology, Integration, and Operations (ATIO) Conference, Arlington, Virginia, 26-28 September, 2005.

${ }^{12}$ Stein, B., Ceniccola, D., and Vincent, D., "TBFM Coupled Scheduling," 56th Annual ATCA Conference and Exposition, National Harbor, Maryland, 2-5 October, 2011 (submitted for publication).

${ }^{13}$ Atkins, S., Jung, Y., Brinton, C., Stell, L., and Rogowski, S., "Surface Management System Field Trial Results," AIAA 2004-6241, AIAA 4th Aircraft Technology, Integration, and Operations Forum, Chicago, Illinois, September 20-22, 2004.

${ }^{14}$ Brinton, C., Lent, S., and Provan, C., "Field Test Results of Collaborative Departure Queue Management," $29^{\text {th }}$ Digital Avionics Systems Conference, Salt Lake City, Utah, 3-7 October 2010.

${ }^{15}$ Hoang, T., Jung, Y., Holbrook, J., and Malik, W., "Tower Controllers' Assessment of the Spot and Runway Departure Advisor (SARDA) Concept," 9th USA/Europe ATM R\&D Seminar (ATM2011), Berlin, Germany, 14-17 June, 2011.

${ }^{16}$ Jung, Y., and Monroe, G., "Development of Surface Management System Integrated with CTAS Arrival Tool," AIAA 2005-7334, AIAA 5th Aviation Technology, Integration, and Operations (ATIO) Conference, Arlington, Virginia, 26-28 September, 2005.

${ }^{17}$ Cook, L., Atkins, S., and Jung Y., "Improved Prediction of Gate Departure Times Using Pre-Departure Events," AIAA 2008-8919, 26th Congress of International Council of the Aeronautical Sciences (ICAS) including the 8th AIAA Aviation Technology, Integration, and Operations (ATIO) Conference, Anchorage, AK, 14-19 September, 2008.

${ }^{18}$ Palopo,K., Chatterji, G., and Lee, H., Benefit Assessment of the Precision Departure Release Capability Concept," AIAA $11^{\text {th }}$ Aviation Technology, Integration, and Operations (ATIO) Conference, Virginia Beach, Virginia, 20-22 September, 2011 (submitted for publication). 